\title{
Casimir interaction between a microscopic dipole oscillator and a macroscopic solenoid
}

\author{
R. Blanco, ${ }^{1}$ K. Dechoum, ${ }^{2}$ H. M. França, ${ }^{2}$ and E. Santos ${ }^{1}$ \\ ${ }^{1}$ Departamento de Fisica Moderna, Universidad de Cantabria, 39005 Santander, Spain \\ ${ }^{2}$ Instituto de Física, Universidade de São Paulo, Caixa Postal 66318, 05315-970, São Paulo, Brazil
}

(Received 16 May 1996; revised manuscript received 9 September 1997)

\begin{abstract}
We discuss the interaction between a microscopic electric dipole oscillator and a long solenoid which are separated by a small distance. The solenoid belongs to a simple $R L C$ circuit and the zero point and thermal current fluctuations within the solenoid coils are taken into account. We describe how they affect the equilibrium state and the excited states of the oscillator, thus providing a description of the Casimir interaction of the system. We calculate the modification in the lifetime of the oscillator excited states as a function of the parameters of the circuit, the dipole orientation, and the distance between the dipole and the solenoid. The Casimir force between the solenoid and the electric dipole is calculated, and it is shown that this Casimir interaction always exists, that is, it occurs even when the macroscopic current in the solenoid is zero. We suggest experiments which can exhibit these effects related to the electromagnetic interactions between atoms or molecules and simple circuits. [S1050-2947(98)00401-6]
\end{abstract}

PACS number(s): 03.65.Sq, 05.40.+j

\section{MOTIVATION AND DESCRIPTION OF THE PHYSICAL SYSTEM}

Two interesting phenomena have motivated us to study the electromagnetic interaction between a macroscopic solenoid and a polarizable molecule or atom. The first one is the Casimir force, which manifests itself between macroscopic objects. This force was predicted theoretically by Casimir [1] in a seminal paper, published in 1948, and entitled "On the Attraction between two Perfectly Conducting Plates." The experimental confirmation of the Casimir prediction was made by Sparnaay [2] in 1958. The Casimir force is nowadays attributed to the existence of fluctuating electromagnetic fields which pervade "empty" space or vacuum. The second phenomenon is the Aharonov-Bohm effect [3] which was also anticipated theoretically in 1959 and confirmed experimentally by Möllenstedt and Bayh [4] in 1962.

We plan to discuss the influence of the current fluctuations on the Aharonov-Bohm effect elsewhere. Nevertheless, motivated by several controversial approaches related to this phenomenon [5], we have decided to use a bound charge to study the dynamical effects of the fluctuating electromagnetic fields in the exterior region of a long solenoid which is part of a simple $R L C$ circuit. It should be noticed, however, that there is already a very large literature describing the perturbative coupling of an oscillator to an electromagnetic cavity [6], but as far as we know, our detailed study of the Casimir interaction between a dipole oscillator and the inductor of the circuit is an original contribution to this field of research.

In order to achieve our goal we shall consider, in detail, the interaction of a long solenoid with an electric dipole placed close to it (a neutral atom or molecule for instance). The electric dipole is a microscopic one-dimensional oscillator (oscillating charge $e$, mass $m$, and frequency $\omega_{0}$ ) which is oriented along the $x$ axis and is placed at the origin of the coordinate system. At a distance $y$ (in the $y$ axis) there is a long solenoid (inductance $L$ ) whose axis is oriented parallel to the $z$ direction. We shall consider that the solenoid has a large number $N$ of circular coils with radius $a$, and that the solenoid extends from $-l / 2$ to $l / 2$ in the $z$ direction ( $L$ $\left.=4 \pi^{2} N^{2} a^{2} / c^{2} l\right)$. We shall also assume that $a \ll l$, that is, the solenoid can be considered thin and very long ("infinite") as far as the interaction with the oscillator is concerned (see Fig. 1).

In order to incorporate the various radiative effects presented by the system, our calculation must be based on quantum electrodynamics (QED) or stochastic electrodynamics (SED) $[7,8]$. It is well known that, for linear systems, the results of SED agree with those of QED [7-10]. For the sake of simplicity our approach will be based on SED, which is very convenient for studying many phenomena associated

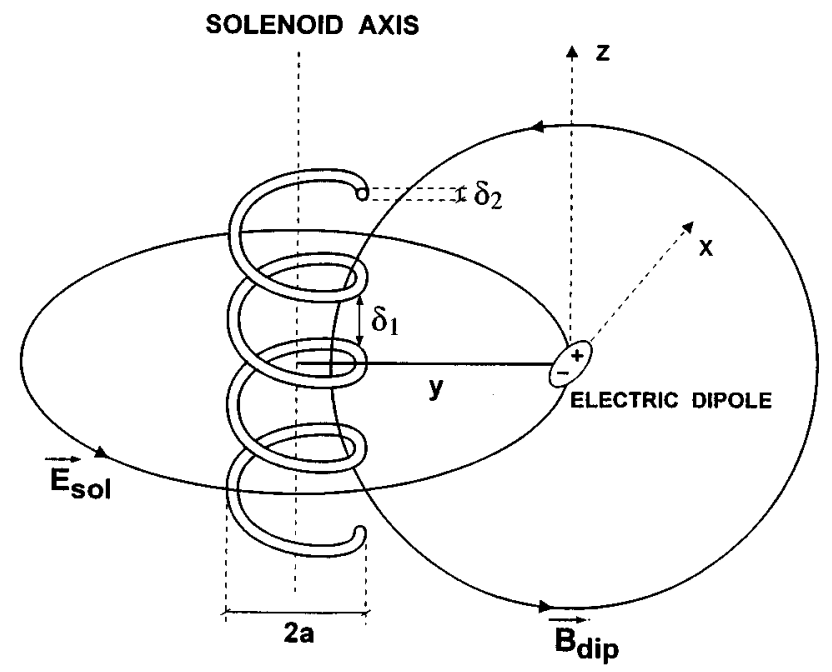

FIG. 1. Schematic picture of the electric dipole at a distance $y$ from the solenoid axis. The relevant fields generated by the solenoid $\left(\vec{E}_{\text {sol }}\right)$ and the oscillating dipole $\left(\vec{B}_{\text {dip }}\right)$ are indicated. The solenoids used by Möllenstedt and Bayh [4] are such that 1.5 $\times 10^{-4} \mathrm{~cm} \leqslant \delta_{1} \leqslant 4 \times 10^{-4} \mathrm{~cm}, \quad \delta_{2} \simeq 3 \times 10^{-4} \mathrm{~cm}, \quad$ and $a \simeq 7$ $\times 10^{-4} \mathrm{~cm}$. The number of coils is $N \simeq 10^{3}$ and the solenoid length $l$ varies from 0.5 to $0.7 \mathrm{~cm}$. 
with electromagnetic fluctuations.

The nonrelativistic equation of motion for the microscopic oscillator is $[7,8]$

$$
m \ddot{x}=-m \omega_{0}^{2} x+e E_{x}(t)+m \tau \ddot{x},
$$

where $\tau=2 e^{2} / 3 m c^{3}$ and $E_{x}(t)$ is the total fluctuating electric field which acts on the charged particle. The last term in Eq. (1.1) is the radiation reaction force which has an important role in SED [9]. Within the dipole approximation (which we shall use throughout this paper) the field $E_{x}(t)$ will be considered only a function of time, independent of the position $x$ of the charge (we are also assuming that $|x| \ll y$ ). In our example there are two contributions to $E_{x}(t)$ which will be denoted by

$$
E_{x}(t)=E_{\mathrm{VF}}(t)+E_{\mathrm{sol}}(t)
$$

where $E_{\mathrm{sol}}(t)$ is the $x$ component of the electric field, generated by the fluctuating current within the solenoid. In Eq. (1.2) $E_{\mathrm{VF}}(t)$ is the $x$ component of the electric field associated with the free space vacuum fluctuations (see Ref. [9]).

In order to facilitate the exposition of our results we shall give, within Sec. II, a brief description of the fluctuations associated with the electric circuit and we also introduce the notation used in our paper. The detailed study of the interaction between the solenoid and the oscillator will be presented in Sec. III. This section will be divided into three parts treating, respectively, the properties of the microscopic oscillator, the Casimir force which the solenoid exerts on the electric dipole, and the modification in the spectral distribution associated with the voltage fluctuations. The possible experimental observation of our predictions and other theoretical implications will be given in Sec. IV.

\section{FLUCTUATIONS ASSOCIATED WITH ELECTRIC CIRCUITS AND WITH THE VACUUM ELECTROMAGNETIC FIELDS}

We shall denote the Fourier transform of $E_{\mathrm{VF}}(t)$ by $\widetilde{E}_{\mathrm{VF}}(\omega)$, that is,

$$
E_{\mathrm{VF}}(t) \equiv \int_{-\infty}^{\infty} d \omega \widetilde{E}_{\mathrm{VF}}(\omega) e^{-i \omega t}
$$

According to the SED approach $[7,8] \widetilde{E}_{\mathrm{VF}}(\omega)$ is a random variable such that its ensemble average $\left\langle\widetilde{E}_{\mathrm{VF}}(\omega)\right\rangle=0$ and

$$
\left\langle\widetilde{E}_{\mathrm{VF}}(\omega) \widetilde{E}_{\mathrm{VF}}\left(\omega^{\prime}\right)\right\rangle=\frac{\hbar \omega^{3}}{3 \pi c^{3}} \operatorname{coth}\left(\frac{\hbar \omega}{2 k T}\right) \delta\left(\omega+\omega^{\prime}\right),
$$

where $\hbar$ is the Planck constant and $T$ is the temperature (zero point and thermal fluctuations are taken into account).

The electric field ( $x$ component) generated by the solenoid at the position of the dipole can be calculated in the standard manner [11]. The retardation effects will be negligible because we shall assume that the relevant frequencies are such that $l \omega \ll c$. Therefore, assuming also $y \ll l$, the result for $E_{\text {sol }}$ acquires a simple familiar expression, namely,

$$
E_{\mathrm{sol}}(t)=-\frac{1}{c} \frac{\partial A_{\mathrm{sol}}}{\partial t} \simeq-\frac{b(y)}{c^{2}} \dot{I}(t),
$$

where $\vec{A}_{\text {sol }}$ is the vector potential, $b(y) \equiv 2 \pi a^{2} N / l y$, and $I(t)$ is the total current within the solenoid coils. We shall discuss first the case in which $\langle I(t)\rangle=\langle\dot{I}(t)\rangle=0$, that is, the circuit is disconnected from a battery.

The correlation function associated with the current fluctuations of the circuit (Nyquist-Johnson noise [12]) is well known in the particular situation in which the electric dipole is very far from the circuit (no interaction). In the case of an $R L C$ circuit we have

$$
\widetilde{I}(\omega)=\frac{\widetilde{\mathcal{E}}(\omega)}{Z(\omega)}
$$

where $Z(\omega)=R-i(\omega L-1 / \omega C)$ is the impedance, and $\widetilde{\mathcal{E}}(\omega)$ and $\widetilde{I}(\omega)$ are, respectively, the Fourier transforms of the random voltage $\mathcal{E}(t)$ and $I(t)$. Assuming that the characteristic wavelength of the emitted radiation $(\lambda=2 \pi c \sqrt{L C})$ is larger than the dimensions of the circuit, that is, neglecting retardation, it is possible to show that $[12,13]$

$$
\left\langle\widetilde{\mathcal{E}}(\omega) \widetilde{\mathcal{E}}\left(\omega^{\prime}\right)\right\rangle=\frac{R \hbar \omega}{2 \pi} \operatorname{coth}\left(\frac{\hbar \omega}{2 k T}\right) \delta\left(\omega+\omega^{\prime}\right) .
$$

The zero point and thermal fluctuations are included in Eq. (2.5). Moreover, we are assuming that the circuit temperature $T$ is equal to the temperature associated with the vacuum electromagnetic field [see Eq. (2.2)]. The result (2.5) follows from the fact that the $R L C$ circuit is quite similar [13] to a harmonic oscillator with frequency $\Omega=(L C)^{-1 / 2}$, that is, the current obeys the equation:

$$
L \dot{I}(t)+R I(t)+\frac{1}{C} \int^{t} I\left(t^{\prime}\right) d t^{\prime}=\mathcal{E}(t),
$$

and the average circuit energy at zero temperature is $\hbar \Omega / 2$ in the limit $R / L \Omega \rightarrow 0$.

The spectral distribution of the vacuum electromagnetic fluctuations [see Eq. (2.2)] will be modified by the presence of the solenoid. We shall calculate this modification under the assumption that the voltage fluctuations and the vacuum electromagnetic fluctuations $E_{\mathrm{VF}}(t)$ are statistically independent, i.e., $\left\langle\widetilde{E}_{\mathrm{VF}}(\omega) \widetilde{\mathcal{E}}\left(\omega^{\prime}\right)\right\rangle=0$. In the absence of interaction (electric dipole removed) the statistical properties of $E_{\text {sol }}$ can be obtained from Eqs. (2.3)-(2.5). In this case the Fourier transform of the total fluctuating electric field, namely, $\widetilde{E_{x}}(\omega) \equiv \widetilde{E_{\mathrm{VF}}}(\omega)+\widetilde{E_{\text {sol }}}(\omega)$, is such that

$$
\left\langle\widetilde{E}_{x}(\omega) \widetilde{E}_{x}\left(\omega^{\prime}\right)\right\rangle=\frac{2 \pi}{3} \rho(\omega, y) \delta\left(\omega+\omega^{\prime}\right),
$$

and is characterized by a spectral distribution $\rho(\omega, y)$. Using Eqs. (2.2)-(2.5) it is possible to show that

$$
\rho(\omega, y)=\frac{\hbar \omega^{3}}{2 \pi^{2} c^{3}} \operatorname{coth}\left(\frac{\hbar \omega}{2 k T}\right)[1+\beta(\omega, y)],
$$

where 


$$
\beta(\omega, y)=\frac{3}{2} \frac{R\left(2 \pi N a^{2} / l y\right)^{2}}{c|Z(\omega)|^{2}} .
$$

The electromagnetic spectral distribution (2.8) is quite different from that observed in free space [see Eq. (2.2)] due to the presence of solenoid. As a matter of fact, the modification in $\rho(\omega, y)$ is significant for frequencies $\omega$ which are close to the circuit frequency $\Omega$. This modification will be essential in order to understand the Casimir interaction between material particles and the solenoid (see Milonni [8] for several other examples of Casimir interactions). This is one of the relevant results of our study. It should be stressed, however, that neither the modification of the spectral density nor the role it plays in the radiative interaction is remotely surprising. This is exactly the essence of the problem as was understood by Purcell [14] in 1946, when he proposed altering the spontaneous radiation rate (at radio frequencies) by coupling magnetic dipoles to a cavity and was abundantly expounded by Barton [15] in his many beautiful papers on the subject.

\section{STUDY OF THE INTERACTION BETWEEN THE SOLENOID AND THE OSCILLATOR}

Let us consider now that the electric dipole is in the origin of the coordinate system at a distance $y$ from the solenoid. The oscillating charge will generate electromagnetic fields which propagate in space and will reach the solenoid (see Fig. 1). Neglecting retardation, the $z$ component of the magnetic field $\vec{B}_{\text {dip }}$ at the point $(0, y, z)$ will be given by [11]

$$
\left(\vec{B}_{\mathrm{dip}}\right)_{z} \simeq \frac{y e \dot{x}(t)}{c r^{3}}
$$

where $r^{2}=y^{2}+z^{2}$ and $x(t)$ is the solution of Eq. (1.1). This time dependent magnetic field will produce an additional fluctuating voltage in each coil of the solenoid because of the fluctuations in $x(t)$. As a consequence, the electromotive force acting in the circuit will be different from $\mathcal{E}(t)$ [see Eqs. (2.5) and (2.6)]. The resulting total electromotive force is

$$
\mathcal{E}_{\mathrm{tot}}(t) \simeq \mathcal{E}(t)+\frac{e}{c^{2}} b(y) \ddot{x}(t),
$$

when retardation effects are negligible (see Ref. [11]). Notice that the geometric factor $b(y)$ is the same factor that appears in Eq. (2.3) which describes the reverse process, that is, the action of the solenoid on the dipole.

In terms of the Fourier transform of $x(t)$, the Eq. (1.1) becomes

$$
\left(\omega_{0}^{2}-\omega^{2}-i \tau \omega^{3}\right) \widetilde{x}(\omega)=\frac{e}{m}\left[\widetilde{E}_{\mathrm{VF}}(\omega)-\frac{i \omega b(y)}{c^{2}} \widetilde{I}(\omega)\right],
$$

where the last term in Eq. (3.3) corresponds to $\widetilde{E}_{\mathrm{sol}}(\omega)$. Equations (2.6) and (3.2) lead to

$$
Z(\omega) \widetilde{I}(\omega)=\widetilde{\mathcal{E}}(\omega)-\frac{e}{c^{2}} \omega^{2} b(y) \widetilde{x}(\omega) .
$$

The above linearly coupled equations, (3.3) and (3.4), can be solved exactly and are the main result of this section. We shall discuss below several new dynamical effects which are governed by Eqs. (3.3) and (3.4).

\section{A. Properties of the microscopic oscillator}

In order to see the new properties of the oscillator, generated by the presence of the cloud of electromagnetic radiation surrounding the solenoid, it is necessary to obtain $\widetilde{x}(\omega)$. From Eqs. (3.3) and (3.4) we get

$$
\widetilde{x}(\omega)=\frac{(e / m)\left[\widetilde{E}_{\mathrm{VF}}-i\left(\omega b / c^{2} Z\right) \widetilde{\mathcal{E}}\right]}{D(\omega)} .
$$

The function $D(\omega)$ introduced above can also be written as

$$
D(\omega)=\omega_{0}^{2}+\tau \omega^{3} \Delta(\omega, y)-\omega^{2}-i \tau \omega^{3}[1+\beta(\omega, y)],
$$

where

$$
\Delta(\omega, y)=\frac{\omega L}{R}\left(1-\frac{\Omega^{2}}{\omega^{2}}\right) \beta(\omega, y)
$$

Notice that the function $\beta(\omega, y)$ is the same function which appears in Eq. (2.8).

In equilibrium, the mean square displacement can be calculated from Eq. (3.5). We shall first show that the familiar result for $\left\langle x^{2}\right\rangle$ is obtained if $\Delta(\omega, y)$ and $\beta(\omega, y)$ are smooth functions of $\omega$ and if $\tau \omega_{0} \ll 1$. According to a standard procedure (see Milonni and the second paper by Boyer in Ref. [8] for a similar calculation) it is possible to show that

$$
\begin{aligned}
\left\langle x^{2}\right\rangle \simeq & \frac{4 \pi}{3} \frac{e^{2}}{m^{2}} \int_{0}^{\infty} d \omega \\
& \times \frac{\left(\hbar \omega^{3} / 2 \pi^{2} c^{3}\right) \operatorname{coth}(\hbar \omega / 2 k T)[1+\beta(\omega, y)]}{\left(\omega^{2}-\omega_{0}^{\prime 2}\right)^{2}+\tau^{2} \omega^{6}[1+\beta(\omega, y)]^{2}} \\
\simeq & \frac{\hbar}{2 m \omega_{0}} \operatorname{coth}\left(\frac{\hbar \omega_{0}}{2 k T}\right),
\end{aligned}
$$

where we have used Eqs. (3.5), (3.6), and (2.8). It is also easy to show that $\omega_{0}^{\prime} / \omega_{0} \simeq 1+\tau \omega_{0} \Delta\left(\omega_{0}, y\right) / 2 \simeq 1$.

The result (3.8) is essentially the free space value for an oscillator in equilibrium with zero point and thermal radiation. This is remarkable because the electric dipole is immersed in the electromagnetic noise generated by the circuit which, however, does not appear in the final expression. The factor $1+\beta$ in the denominator of the integral (3.8) counterbalances the factor $1+\beta$ which appears in the spectral distribution $\rho(\omega, y)$. A simple consequence of this fact is that the average value of the oscillator energy at $T>0$ is precisely $(1 / 2) \hbar \omega_{0} \operatorname{coth}\left(\hbar \omega_{0} / 2 k T\right)$. This is, therefore, a striking example of the fluctuation-dissipation mechanism in SED. Another impressive example of this fluctuation-dissipation mechanism, associated with coupled mechanical oscillators, was considered by Blanco et al. [16] in their "Classical interpretation of the Debye law for the specific heat of solids." 
We are going to study now the response of the oscillator to a voltage excitation applied to the circuit. We shall assume that it is generated by a deterministic disturbance $V_{d}(t)$ in the circuit voltage and we shall describe it replacing $\widetilde{\mathcal{E}}(\omega)$ by $\widetilde{\mathcal{E}}(\omega)+\widetilde{V}_{d}(\omega)$ in Eq. (3.5). For simplicity we shall assume that $\widetilde{V}_{d}(\omega)$ has a constant value $A_{0} / 2 \pi$. This hypothesis corresponds to generating a deterministic voltage pulse into the circuit which has the simple form $V_{d}(t)=A_{0} \delta(t)$. Therefore according to Eq. (3.5) the average position of the oscillator will be given by

$$
\begin{aligned}
\langle x\rangle= & \frac{e}{m} \int_{-\infty}^{\infty} d \omega e^{-i \omega t} \frac{(-i \omega b)\left(A_{0} / 2 \pi\right)}{c^{2} Z(\omega) D(\omega)} \\
\simeq & \frac{2 \pi N a^{2}}{l y} \frac{A_{0}}{c^{2} L} \frac{e / m}{\rho_{+} \rho_{-}}\left[-\Omega \sin (\Omega t) \exp \left(-\frac{R}{2 L} t\right)\right. \\
& \left.+\omega_{0} \sin \left(\omega_{0} t\right) \exp \left(-\frac{\Gamma^{\prime}}{2} t\right)\right]
\end{aligned}
$$

where $\rho_{ \pm} \equiv \sqrt{\left(\Omega \pm \omega_{0}\right)^{2}+\left[(R / 2 L)-\Gamma^{\prime} / 2\right]^{2}}$ and $\Gamma^{\prime}=\Gamma[1$ $\left.+\beta\left(\omega_{0}, y\right)\right]$. Notice that $\Gamma \equiv 2 e^{2} \omega_{0}^{2} / 3 m c^{3}$ is the damping constant in free space. The above result was obtained by assuming that $\Gamma^{\prime} \ll \omega_{0}, R / L \ll \Omega$ and that the poles in the integrand are $\pm \omega_{0}-i \Gamma^{\prime} / 2$ and $\pm \Omega-i R / 2 L$.

An interesting remark is that the damping constants in both terms in Eq. (3.9) are different from the free space damping constant $\Gamma$ due to the presence of the solenoid. According to Eq. (3.9) we see that the lifetime of the oscillator states can be "controlled" by the experimentalist who can modify the parameters associated with the circuit.

To have a qualitative idea of the lifetime associated with the first term in Eq. (3.9), as compared with the free space value, let us assume that $\omega_{0} \simeq 10^{11} \mathrm{sec}^{-1}$ and $\tau \omega_{0} \simeq 10^{-16}$. These two values are characteristic of the oscillations associated with a simple molecule such as the $\mathrm{NH}_{3}$, for instance. Therefore, using the numerical values for $R$ and $L$ considered in Ref. [4], namely $R \simeq 4 \times 10^{-10} \mathrm{sec} / \mathrm{cm}$ and $L \simeq 5$ $\times 10^{-20} \mathrm{sec}^{2} / \mathrm{cm}$, we get $R / L \Gamma=R / L \tau \omega_{0}^{2} \simeq 10^{15}$. Such a huge value, obtained in this particular example, implies that the radiation emitted by the electric dipole will have a broad spectral distribution in comparison with the free space case. In our opinion, this theoretical prediction is quite interesting and deserves further attention from the experimental point of view. The anisotropy in the radiation emitted by the oscillator may be experimentally detected. It is also possible to show that $\Gamma^{\prime} / \Gamma \simeq 1+10 a^{2} / y^{2}$. Therefore, the result (3.9) shows that we have an enhancement in the spontaneous emission by the oscillator. A similar situation is encountered when the oscillator is between two parallel mirrors $[17,18]$.

\section{B. Casimir force}

The electromagnetic force (nonrelativistic Lorentz force) which the solenoid exerts on the microscopic electric dipole $\vec{p}(t)$ is given by the ensemble average (see Ref. [19])

$$
\vec{F} \simeq\left\langle(\vec{p} \cdot \vec{\nabla}) \vec{E}_{\mathrm{sol}}\right\rangle+\left\langle\frac{\vec{p}}{c} \times \vec{B}_{\mathrm{sol}}\right\rangle
$$

The electric dipole has only the component $p_{x}(t) \equiv \operatorname{ex}(t)$ and $x(t)$ has the Fourier transform given by Eq. (3.5). The solenoid electric field $\vec{E}_{\text {sol }}(t)$ was obtained before and its $x$ component is given by Eq. (2.3). The magnetic field $\vec{B}_{\text {sol }}=\vec{\nabla}$ $\times \vec{A}_{\text {sol }}$ is in the $z$ direction and one can show that it gives a negligible contribution to $\vec{F}$ despite the fact that $\left|\vec{B}_{\text {sol }}\right|$ $=\left|\vec{E}_{\text {sol }}\right|$.

The force (3.10) is in the $y$ direction and can be written as

$$
F \equiv\left\langle\left[(\vec{p} . \vec{\nabla}) \vec{E}_{\mathrm{sol}}\right]_{y}\right\rangle=2 \pi \frac{a^{2} N}{c^{2} l y^{2}} e\langle x \dot{I}\rangle
$$

The current $I(t)$ has a Fourier transform $\widetilde{I}(\omega)$ such that

$$
Z(\omega) \widetilde{I}(\omega)=\frac{\omega_{0}^{2}-\omega^{2}-i \tau \omega^{3}}{D(\omega)} \widetilde{\mathcal{E}}(\omega)-\frac{3}{2} \frac{\tau \omega^{2} c b(y)}{D(\omega)} \widetilde{E}_{\mathrm{VF}}(\omega)
$$

in agreement with the coupled Eqs. (3.3) and (3.4). Taking into account Eqs. (3.5), (3.12), (2.2), and (2.5) it is possible to show that $F$ can be expressed as the integral

$$
\begin{aligned}
F= & -3 \pi\left(\tau \omega_{0}\right)\left(\hbar \omega_{0}\right) \frac{N^{2} a^{4}}{l^{2} y^{3}}(c R) \\
& \times \int_{-\omega_{\max }}^{\omega_{\max }} d \omega \frac{\omega^{3} \operatorname{coth}(\hbar \omega / 2 k T)}{\omega_{0}^{2}|c Z(\omega)|^{2}|D(\omega)|^{2}} \\
& \times\left[\left(\omega_{0}^{2}-\omega^{2}\right)+\tau \omega \frac{L \omega}{R}\left(\Omega^{2}-\omega^{2}\right)\right],
\end{aligned}
$$

where $\omega_{\max }$ is the maximum frequency compatible with the long wavelength approximation used within this paper $\left(\omega_{\max } \simeq c / l\right)$.

The integral in expression (3.13) can be calculated for any value of the temperature $T$. We shall comment first on the result for $k T \ll \hbar \Omega$ and $k T \ll \hbar \omega_{0}$ which can be obtained from Eq. (3.13) by replacing $\omega^{3} \operatorname{coth}(\hbar \omega / 2 k T)$ by $|\omega|^{3}$. In this case the result is

$$
\begin{aligned}
F= & -\epsilon \frac{3}{4 \pi} \frac{a^{2}}{y^{3}}\left(\frac{c \hbar}{l}\right)\left\{2 \gamma \epsilon^{\prime} \ln \left(\frac{c}{l \omega_{0}}\right)+\frac{\pi\left(1-\gamma^{2}\right)}{\left(1-\gamma^{2}\right)^{2}+\epsilon^{\prime 2} \gamma^{2}}\right. \\
& \left.+\frac{2 \gamma^{3} \epsilon^{\prime}\left(2-\gamma^{2}-\epsilon^{\prime 2}\right) \ln \gamma+4 \gamma^{3}\left[\gamma^{2}\left(1-\epsilon^{\prime 2} / 2\right)-\left(\epsilon^{\prime 4}-4 \epsilon^{\prime 2}+2\right) / 2\right] \psi\left(\epsilon^{\prime}\right)}{\left(1-\gamma^{2}\right)^{2}+\epsilon^{\prime 2} \gamma^{2}}\right\},
\end{aligned}
$$


where $\psi\left(\epsilon^{\prime}\right) \equiv\left(4-\epsilon^{\prime 2}\right)^{-1 / 2} \arctan \left(\sqrt{4-\epsilon^{\prime 2}} / \epsilon^{\prime}\right)$. The dimensionless parameters $\epsilon, \epsilon^{\prime}$, and $\gamma$ are defined by

$$
\epsilon \equiv \tau \omega_{0}, \quad \epsilon^{\prime} \equiv \frac{R}{L \Omega}, \quad \text { and } \gamma \equiv \frac{\Omega}{\omega_{0}} .
$$

The result (3.14) is valid up to first order in $\epsilon$ and provided that $\omega_{0}$ and $\Omega$ are much less than $c / l$. See the Appendix for the calculation details.

In the particular case $\epsilon^{\prime} \ll 1$, the expression within the curly brackets in Eq. (3.14) acquires a very simple form, namely, $\pi\left[1+\gamma^{2} /(1+\gamma)\right]$. It is remarkable that this is true for $|1-\gamma| \gg \epsilon^{\prime}$ and $|1-\gamma| \simeq \epsilon^{\prime}$.

Another interesting case occurs when the temperature is such that $k T \gg \hbar \omega_{\max }$. One can, therefore, replace $\hbar \omega \operatorname{coth}(\hbar \omega / 2 k T)$ by $k T$ in Eq. (3.13) and the Casimir force will be given by a much simpler expression in comparison with Eq. (3.14), namely,

$$
F=-\frac{3}{4} \frac{\tau c a^{2}}{l y^{3}} k T .
$$

According to this result the force is repulsive, increases linearly with the temperature, and is independent of the oscillator frequency $\omega_{0}$ provided that $\omega_{0} \ll \omega_{\max }=\mathrm{c} / \mathrm{l}$.

Finally, if $\omega_{0} \gg \omega_{\max }$, the force calculated from Eq. (3.13) becomes very small.

\section{Spectral density of the voltage fluctuations in the $R L C$ circuit}

According to Eq. (3.2) the spontaneous fluctuation of the total electromotive force $\mathcal{E}_{\text {tot }}(t)$ acting in the circuit has two main contributions. The first one, indicated by $\mathcal{E}(t)$ in Eq. (3.2), is simply the thermal and zero point noise voltage in the resistive circuit $[12,13]$. Its spectral density is proportional to the resistance $R$ of the circuit [see Eq. (2.5)]. The second one is generated by the fluctuations of the electric dipole and is proportional to $\ddot{x}(t)$.

The Fourier transform of $\mathcal{E}_{\text {tot }}(t)$ has a spectral density $S_{V}(\omega)$ which can be written in a standard form, namely, $\left\langle\widetilde{\mathcal{E}}_{\text {tot }}(\omega) \widetilde{\mathcal{E}}_{\text {tot }}\left(\omega^{\prime}\right)\right\rangle=S_{V}(\omega) \delta\left(\omega+\omega^{\prime}\right)$. The two contributions to $S_{V}(\omega)$ can be easily obtained from our previous equations (2.5), (3.2), and (3.5). Therefore it is straightforward to show that

$$
\begin{aligned}
S_{V}(\omega) \simeq & \frac{\hbar \omega}{2 \pi} \operatorname{coth}\left(\frac{\hbar \omega}{2 k T}\right)\left\{R+\frac{(\tau \omega)^{2}}{c} \frac{3}{2}\left(\frac{2 \pi a^{2} N}{l y}\right)^{2}\right. \\
& \left.\times \frac{\omega^{4}[1+\beta(\omega, y)]}{\left(\omega_{0}^{2}-\omega^{2}\right)^{2}+\tau^{2} \omega^{6}[1+\beta(\omega, y)]^{2}}\right\}
\end{aligned}
$$

where $\beta(\omega, y)$ is given by Eq. (2.9).

The second term in Eq. (3.17) is caused by the fluctuating magnetic field generated by the random oscillations of the charge in the electric dipole [see Eq. (3.1)]. This contribution to the spectral density $S_{V}(\omega)$ is small, in a wide range of the spectrum, due to the factor $(\tau \omega)^{2}$. However, it should be mentioned that, for frequencies very close to $\omega_{0}$, the second term in Eq. (3.17) has a very sharp peak which is of zero order in $\tau$. The possible experimental observation of this sharp peak will be discussed in the following section.

\section{DISCUSSION}

An experimental test of our prediction for Casimir force, Eqs. (3.14) and (3.16), might be performed measuring the deviation of an appropriate molecule passing near the solenoid. A similar procedure has been used for the measurement of the Casimir-Polder force on atoms passing through a micrometer-sized parallel-plate cavity [20]. In our case, we should use a molecule with a characteristic vibration of frequency $\omega_{0}$ smaller than $c / l$.

A good candidate is the inversion of the ammonia molecule, whose frequency correspond to a radiation wavelength $\lambda=1.3 \mathrm{~cm}$. However, the Casimir force in these conditions is extremely small and is probably beyond the capabilities of present-day technology. Using Eq. (3.16) for room temperature, we have estimated the angle $\alpha$ of deviation of a molecule passing close $(y \simeq a)$ to the solenoid to be

$$
\alpha \simeq \frac{\pi}{8} \frac{\tau c}{l} \frac{k T}{M v^{2}} \simeq 5 \times 10^{-8} v^{-2},
$$

where $v$ is the velocity in $\mathrm{cm} / \mathrm{sec}$. We have taken $\tau$ $=(2 / 3)\left(e^{2} / m c^{3}\right) \simeq 10^{-27}$ sec because $e$ is the proton charge and $m$ the reduced mass of the $\mathrm{NH}_{3}$ molecule (about three times the proton mass). Moreover, $M$ was assumed to be the mass of the entire molecule (17 times the proton mass).

It is interesting that Eq. (4.1) gives a deviation which is inversely proportional to the mass and does not depend on the vibration frequency ( except that $\omega_{0} \ll c / l$ and $\hbar \omega_{0}$ $\ll k T)$. This suggests that the Casimir force between a free electron and a solenoid might give a measurable deviation, much bigger than Eq. (4.1). The experiment would be similar to and no more difficult than the standard experimental test of the Aharonov-Bohm effect [3-5]. A calculation of the Casimir force between a solenoid and a free electron is in progress.

Another prediction that can be tested experimentally derives from Eq. (3.17), namely, the measurement of the effect of the dipole oscillator on the voltage (or the current) fluctuations in the $R L C$ circuit. Some of these fluctuations have already been measured [12]. As the product $\tau \omega_{0}$ is extremely small, we may write Eq. (3.17) in the simplified form

$$
\begin{aligned}
S_{V}(\omega) \simeq & \frac{\hbar \omega}{2 \pi} \operatorname{coth}\left(\frac{\hbar \omega}{2 k T}\right) L \Omega \\
& \times\left\{\epsilon^{\prime}+\frac{3 \pi}{4}\left(\tau \omega_{0}\right) \frac{\omega_{0} c a^{2}}{\Omega l y^{2}} \delta\left(\omega-\omega_{0}\right)\right\},
\end{aligned}
$$

where $\delta$ is Dirac's delta. We predict a very sharp peak in the spectrum at $\omega=\omega_{0}$ but the width and height of the peak will be determined by the resolution $(\Delta \omega)$ in the measurement of the frequency. Averaging the terms of Eq. (4.2) that are within curly brackets, in the interval $\left[\omega_{0}-(\Delta \omega) / 2\right.$, $\left.\omega_{0}+(\Delta \omega) / 2\right]$ we get

$$
\epsilon^{\prime}+\frac{3 \pi}{4}\left(\tau \omega_{0}\right) \frac{\omega_{0}}{(\Delta \omega)} \frac{c}{\Omega l} \frac{a^{2}}{y^{2}},
$$


if $\omega$ lies inside the interval. Even in the closest approach of the oscillator to the solenoid (i.e., $y \simeq a$ ) we get for the second term of Eq. (4.3) a value of about $10^{-14}$ to be compared with $\epsilon^{\prime}(\simeq 1 / 20)$ using $(\Delta \omega) / \omega_{0} \simeq 10^{-2}$, and the parameters of the Möllenstedt and Bayh solenoid plus an ammonia molecule. This is too small to be measurable. Nevertheless, we may consider a large number of molecules by immersing the solenoid in a cylinder of radius $l$ and height $l$ filled with ammonia gas. In this case, a straightforward calculation shows that the second term of Eq. (4.3) should be multiplied by

$$
\frac{2 \pi}{3} n a^{2} l \ln \left(\frac{l}{a}\right)
$$

where $n$ is the number of molecules per unit volume (2.7 $\times 10^{19} \mathrm{~cm}^{-3}$ at room temperature). We predict a pronounced peak (about five times bigger than the background) when $\omega$ approaches the ammonia inversion frequency.

As a final remark we want to suggest that the above results (Secs. III C and IV) may be useful in the study of "stochastic resonance" effects. These phenomena [21], observed in various nonlinear systems including electronic circuits, are characterized by the presence of an (optimal) noise amplitude which can enhance the detection of weak periodic signals. Therefore we believe that our analysis may also find useful application in this wide context.

\section{ACKNOWLEDGMENTS}

We acknowledge financial support from Caja Cantabria, DGCYT Project Number PB 92-0507 (Spain), Conselho Nacional de Desenvolvimento Científico e Tecnológico ( $\mathrm{CNPq}$ Brazil), and Fundação de Amparo à Pesquisa do Estado de São Paulo (FAPESP). We also thank A. M. Cetto, C. P. Malta, T. W. Marshall, and L. de la Peña for valuable comments.

\section{APPENDIX}

We give below a brief description of the method we have used in order to obtain the result (3.14) for the Casimir force at zero temperature. The calculation of the mean square displacement $\left\langle x^{2}\right\rangle$, of the average oscillator energy, and also the calculation of the Casimir force in the high temperature limit $\left(k T / \hbar \gg \omega_{\max }=c / l\right)$, are similar.

Introducing the variable $x=\omega / \omega_{0}$ the expression (3.13) will be given by

$$
F=-\frac{3}{2 \pi}\left(\frac{a^{2}}{y^{3}}\right)\left(\frac{c}{l \omega_{0}}\right) \hbar \omega_{0} \epsilon I,
$$

where

$$
\begin{aligned}
I & \equiv \int_{0}^{c / \omega_{0} l} d x \frac{P(x)}{\mathcal{D}_{\epsilon}(x)} \\
& \equiv \int_{0}^{c / \omega_{0} l} d x\left[\frac{\epsilon x^{7}\left(x^{2}-\gamma^{2}\right)+\epsilon^{\prime} \gamma x^{5}\left(x^{2}-1\right)}{\mathcal{D}_{\epsilon}(x)}\right]
\end{aligned}
$$

the function $\mathcal{D}_{\epsilon}(x)$ being

$$
\begin{aligned}
& \mathcal{D}_{\epsilon}(x)=\left[\left(x^{2}-\gamma^{2}\right)^{2}+\epsilon^{\prime 2} \gamma^{2} x^{2}\right]\left[\left(1-x^{2}\right)^{2}+\epsilon^{2} x^{6}\right] \\
& +2 \chi \epsilon x^{4}\left(1-x^{2}\right)\left(x^{2}-\gamma^{2}\right)+\epsilon^{2}\left(2 \chi \gamma \epsilon^{\prime}+\chi^{2}\right) x^{8} .
\end{aligned}
$$

The parameters $\epsilon, \epsilon^{\prime}$, and $\gamma$ were defined in Eq. (3.15) and $\chi=3 a^{2} c / 2 y^{2} l \omega_{0}$.

The ratio $P(x) / \mathcal{D}_{\epsilon}(x)$ can be written in a form which is convenient for the integration in Eq. (A2), namely,

$$
\frac{P(x)}{\mathcal{D}_{\epsilon}(x)}=\frac{A x}{\epsilon^{2} x^{2}+\rho^{2}}+\left(\sum_{j=1}^{4} \frac{\alpha_{j}}{x-Z_{j}}+\text { c.c. }\right)
$$

The constants $A, \rho, \alpha_{j}$, and $Z_{j}$ will be calculated only up to first order in $\epsilon=\tau \omega_{0} \ll 1$. Notice that we shall assume that $\omega_{\max }=c / l \ll 1 / \tau$. One can show that

$$
A=\epsilon\left(1-\frac{\epsilon \epsilon^{\prime} \gamma\left[1-\gamma^{2}\left(2-\epsilon^{\prime 2}-\gamma^{2}\right)\right]}{\zeta}\right)+O\left(\epsilon^{3}\right),
$$

where $\zeta=\left(1-\gamma^{2}\right)^{2}+\gamma^{2} \epsilon^{\prime 2}$, and $\rho=1-\chi \epsilon+O\left(\epsilon^{2}\right)$.

Moreover, we have

$$
\alpha_{j}=\frac{P\left(Z_{j}\right)}{\mathcal{D}_{\epsilon}^{\prime}\left(Z_{j}\right)}
$$

with $j=1,2,3,4$ and the values of $Z_{j}$ are

$$
Z_{1} \equiv-Z_{2}=1+\epsilon \frac{\chi\left(1-\gamma^{2}\right)}{2 \zeta}+\frac{i}{2} \epsilon\left(1+\frac{\chi \gamma \epsilon^{\prime}}{\zeta}\right)+O\left(\epsilon^{2}\right)
$$

and

$$
Z_{3} \equiv-Z_{4}=\gamma \sqrt{1-\frac{\epsilon^{\prime 2}}{4}}+\frac{i}{2} \epsilon^{\prime} \gamma+O(\epsilon)
$$

The substitution of Eq. (A4) into Eqs. (A2) and (A1) leads to the result (3.14) for the Casimir force.
[1] H. B. G. Casimir, Proc. K. Ned. Akad. Wet. 51, 360 (1948); H. B. G. Casimir, and D. Polder, Phys. Rev. 73, 360 (1948).

[2] M. J. Sparnaay, Physica (Amsterdam) 24, 751 (1958). See also S. K. Lamoreaux, Phys. Rev. Lett. 78, 5 (1997), for a remark- able measurement of the Casimir force.

[3] Y. Aharonov and D. Bohm, Phys. Rev. 115, 485 (1959).

[4] G. Möllenstedt and W. Bayh, Naturwissenschaften 49, 81 (1962); W. Bayh, Z. Phys. 169, 492 (1962). 
[5] S. Olariu and J. J. Popescu, Rev. Mod. Phys. 57, 339 (1985); M. Peshkin and A. Tonomura, The Aharonov-Bohm Effect (Springer-Verlag, Berlin, 1989). See also, T. H. Boyer, Phys. Rev. D 8, 1667 (1973); 8, 1679 (1973); Nuovo Cimento B 100, 685 (1987).

[6] See the various papers which appear in Cavity Quantum Electrodynamics, edited by Paul R. Berman, special issue of Adv. At. Mol. Opt. Phys. Suppl. 2 (1994).

[7] L. de la Peña and Ana Maria Cetto, The Quantum Dice. An Introduction to Stochastic Electrodynamics, edited by A. van der Merwe (Kluwer Academic Publishers, Dordrecht, 1996).

[8] T. H. Boyer, Phys. Rev. D 11, 790 (1975); 11, 809 (1975). See also P. W. Milonni, The Quantum Vacuum: An Introduction to Quantum Electrodynamics (Academic Press, Inc. Boston, 1994).

[9] H. M. França and T. W. Marshall, Phys. Rev. A 38, 3258 (1988). See also K. Dechoum and H. M. França, Found. Phys. 25, 1599 (1995); 26, 1573 (1996). These papers give a detailed discussion of the combined effects of the vacuum electromagnetic fluctuations and radiation reaction on the time evolution of the physical and nonphysical states of the harmonic oscillator.

[10] R. Schiller and H. Tesser, Phys. Rev. A 3, 2035 (1971). See also J. Dalibard, J. Dupond-Roc, and C. Cohen-Tannoudji, J. Phys. (Paris) 43, 1617 (1982).

[11] J. D. Jackson, Classical Electrodynamics, 2nd ed. (John Wiley and Sons, New York, 1975), Chap. 9, Sec. 9.3. See also R. V. Sampaio and R. N. Suave, Am. J. Phys. 64, 300 (1996).
[12] V. L. Ginzburg, Applications of Electromagnetism in Theoretical Physics and Astrophysics (Gordon and Breach Science Publishers, New York, 1989), Chap. 14. A clear observation of zero point fluctuations in a resistively shunted Josephson tunnel junction was reported by R. H. Koch, D. J. Van Harlingen, and J. Clarke, Phys. Rev. Lett. 47, 1216 (1981). See also G. Y. Hu and R. F. O'Connell, Phys. Rev. B 46, 14219 (1992) for other experimental observations of Nyquist noise and zero point fluctuations.

[13] J. Weber, Phys. Rev. 90, 977 (1953); 94, 211 (1954). See also D. A. Bell, Electrical Noise (D. Van Nostrand Company Ltd., London, 1960).

[14] E. M. Purcell, Phys. Rev. 69, 681 (1946).

[15] See the paper by G. Barton quoted in [6], and references therein.

[16] R. Blanco, H. M. França, and E. Santos, Phys. Rev. A 43, 693 (1991).

[17] H. M. França, T. W. Marshall, and E. Santos, Phys. Rev. A 45, 6436 (1992). See also A. M. Cetto and L. de la Peña, ibid. 37, 1952 (1988); 37, 1960 (1988); and the interesting review by S. Haroche and J. M. Raimond, Sci. Am. (Int. Ed.) 268(4), 54 (1993).

[18] See the paper by E. A. Hinds in our Ref. [6].

[19] T. H. Boyer, Phys. Rev. A 7, 1832 (1973).

[20] C. I. Sukenik, M. G. Boshier, D. Cho, V. Sondoghdar, and E. A. Hinds, Phys. Rev. Lett. 70, 560 (1993).

[21] K. Wiesenfeld and F. Moss, Nature (London) 373, 33 (1995). 